\title{
Diagnosis and management of primary pyogenic spinal infections in intravenous recreational drug users
}

\author{
Mateo ZiU, M.D., ${ }^{1,2}$ Bradley Dengler, M.D., ${ }^{2}$ Davin Cordell, M.D., ${ }^{2}$ \\ and Viktor Bartanusz, M.D., Рh.D. ${ }^{2}$
}

${ }^{1}$ Seton Brain and Spine Institute, Austin; and ${ }^{2}$ Department of Neurosurgery, University of Texas Health Science Center in San Antonio, Texas

\begin{abstract}
Object. Primary spine infection secondary to intravenous drug abuse (IVDA) is a difficult clinical entity encountered by spine surgeons and infectious disease specialists. Patients tend to be noncompliant with the treatment and follow-up, and some continue to use IV recreational drugs even after the diagnosis of spine infection. The authors undertook this study to analyze the presentation, etiology, demographic characteristics, treatment, and outcome of primary pyogenic spinal infection in patients with IVDA as the major risk factor.

Methods. The medical records, radiology imaging, and laboratory results (white blood cell count, inflammatory markers, bacteriology cultures) of all patients with pyogenic spine infection and history of IVDA presenting to a tertiary care center from August 2005 through December 2013 were retrospectively reviewed. The department of neurosurgery database and the hospital electronic medical records of University Hospital in San Antonio were used to identify the cohort for our study.

Results. A total of 164 patients with spinal infection were evaluated during the study period; 102 of these patients had a history of IVDA. Their average age was 45.4 years, and only $14(13.7 \%)$ were women. The mean laboratory values at presentation included a white blood cell count of $11.1 \times 10^{3}$ cells/ $\mu \mathrm{l}$ (range $0.5-32 \times 10^{3}$ cells/ $\mu \mathrm{l}$ ), erythrocyte sedimentation rate (ESR) of $74 \mathrm{~mm} / \mathrm{hr}$ (range $9.9-140 \mathrm{~mm} / \mathrm{hr}$ ), and C-reactive protein (CRP) level of $67 \mathrm{mg} / \mathrm{L}$ (range $0.1-327 \mathrm{mg} / \mathrm{L}$ ). Twenty-six patients $(25.4 \%)$ had an associated epidural abscess. The most common organism isolated from cultures of the bone and/or blood was methicillin-sensitive Staphylococcus aureus (MSSA), which was found in 37 cases. A close second was methicillin-resistant S. aureus (MRSA), found in 23 cases. The most commonly involved region was the lumbar spine (24 cases [57.8\%]), and most patients $(69.6 \%)$ had involvement of only a single level. Eighty patients were initially treated with long-term IV antibiotic therapy, and only 22 underwent surgical intervention ( 24 procedures). Of the latter group, 8 patients underwent laminectomy alone while 16 required some type of instrumented stabilization. Of the patients requiring stabilization procedures, 2 (12.5\%) required reoperation with extension of their surgical constructs to other levels. The average follow-up was 29.7 weeks (range 6 weeks to 3 years).

Conclusions. Diagnosis and management of spinal infection in patients with a history of IVDA is challenging. The data from this study show that initial laboratory values are difficult to interpret given that only a minority of these patients present with leukocytosis. Back pain was the only reliable predictor of spine infection. The authors' experience indicates that the majority of patients with spine infection and a history of IVDA can be successfully treated with IV antibiotic therapy alone.
\end{abstract}

(http://thejns.org/doi/abs/10.3171/2014.6.FOCUS14148)



$\mathrm{P}$ RIMARY spinal infections are a significant clinical problem with a substantial financial burden and impact on the quality of life of the patient..$^{16}$ Their insidious presentation and slow clinical course make the diagnosis and subsequent treatment difficult. Due to the inconsistent presentation of these entities, delay in diagnosis is not infrequent. This can result in suppuration into

\footnotetext{
Abbreviations used in this paper: AIS = ASIA Impairment Scale; ASIA $=$ American Spinal Injury Association; CRP $=$ C-reactive protein; $\mathrm{ESR}=$ erythrocyte sedimentation rate; IVDA = intravenous drug abuse; MRSA = methicillin-resistant Staphylococcus aureus; MSSA = methicillin-sensitive $S$. aureus; $\mathrm{WBC}=$ white blood cell .
}

the spinal canal with spinal cord compression, leading to permanent neurological deficit or even death. The incidence of vertebral infection is about 2.2 per $100,000 .{ }^{16}$ It occurs predominately in the 5th decade of life. Although once it was regarded as a rare event, in recent years there has been an increase in the reported number of patients presenting with primary pyogenic infection of the spine..$^{1,2,4,10,11,17}$ The reasons are multifactorial and have been attributed for the most part to the increase in intravenous drug abuse (IVDA) and the increase in life expectancy of older patients and those with chronic debilitating diseases..$^{12,17}$ Other predisposing factors for pyogenic spinal infections include malnutrition, compromise 
of the immune system, diabetes mellitus, HIV positivity, malignancy, chronic steroid usage, renal failure, septicemia, recent spinal surgery, and intravascular devices. ${ }^{2}$

Vertebral infection has remained a challenging problem despite advances in medical knowledge, imaging techniques, and surgical spine instrumentation. ${ }^{8}$ There is no consensus regarding its optimal management or the optimal duration of parenteral antibiotic therapy and of subsequent oral therapy. ${ }^{13}$ Furthermore, questions remain regarding whether and when to obtain tissue cultures from the infected vertebra in cases in which blood cultures are positive, since it is known that the latter are accurate in identification of the pathogen involved in the spinal infection in approximately $85 \%$ of cases..$^{14,15}$ Nevertheless, in the majority of cases of primary spinal infections, conservative management of infection with antibiotic therapy is successful. ${ }^{5}$ However, in a minority of cases, surgical intervention is warranted.

As mentioned above, one of the main reasons for the increase in the incidence of spinal infections is thought to be the increase in the rate of recreational drug abuse. Unfortunately, diagnosis and treatment of these pathological conditions in this population is challenging. Many of these patients experience irreversible neurological deficits due to their late presentation for medical attention, the lack of adequate follow-up, continuation of their drug abuse habits, and the need for prolonged hospitalization to prevent them from using the intravenous access devices other than for antibiotic injection. ${ }^{19}$ It is estimated that the mean annual health cost per IVDA patient diagnosed with soft-tissue or skeletal infection is CAD $\$ 119,574 .{ }^{19}$ Unfortunately, protocols for diagnosis and management of spine infections in the IVDA population are lacking.

Here we retrospectively reviewed 102 cases of primary spine infection in patients with a history of IVDA. The purpose of our review was to define the contemporary trends in etiology, concomitant risk factors, presentation, and management of spine infection in this patient population. Additionally, we determine factors leading to surgical intervention and describe the outcomes.

\section{Methods}

We initially identified all cases involving patients evaluated in our institution (University Hospital, San Antonio, Texas) for spine infection during the period from August 2005 through December 2013. From these cases, those involving pyogenic spinal infection in patients with a history of recreational IVDA were selected for this retrospective review. All cases of postsurgical wound infection, so-called secondary spine infection, were excluded, unless the first surgical intervention was performed for spine infection. The data extracted from the electronic medical records of University Hospital and the neurosurgery department database included patient age, sex, site of infection, concomitant diseases, risk factors, preceding events, clinical presentation, laboratory values, treatment modalities, surgical indications, type of procedure performed, and outcomes. The following laboratory data were reviewed: erythrocyte sedimentation rate (ESR), white blood cell (WBC) count, and C-reactive protein
(CRP) level. Microbiological results from blood, urine, and spine biopsy cultures as well as any other tissue cultures were reviewed. Radiological studies reviewed included information from plain radiographs, CT, and MRI on admission and follow-up.

Diagnosis was made on the basis of a combination of clinical history, physical examination findings, results of radiological assessment, and positive organism cultures. As previously described, ${ }^{2}$ diagnosis of spinal infection was additionally given in cases with suggestive clinical features and appropriate radiological changes, in which microbial cultures remained negative, but the patients had elevated inflammatory markers and/or responded favorably to antimicrobial therapy. All surgical reports for the surgically treated patients were reviewed with emphasis on the type of approach, need for reoperation, and the number of levels instrumented.

This study was approved by the institutional review board of the University of Texas Health Science Center at San Antonio and University Hospital in San Antonio, Texas.

\section{Results}

\section{Demographics}

One hundred sixty-four patients with primary pyogenic spinal infection were treated at our institution between August 2005 and December 2013. From these, 102 patients (62\%) with a history of remote or current recreational IVDA were identified. Most of the patients were male (87 men [85.3\%], 14 women [13.7\%], ratio of 5.8:1). Their mean age at presentation was 45.4 years, with the majority being in the 41 - to 60 -year-old age group (range 22-66 years) (Table 1).

\section{Diagnosis and Presentation}

All 102 patients presented with persistent neck or back pain, depending on the level of the disease. MRI showed findings consistent with spondylodiscitis with or without epidural abscess, exclusive epidural abscess, or spondylodiscitis expanding into the surrounding paravertebral soft tissue. Seven patients $(6.9 \%)$ presented with epidural abscess alone, 19 (18.6\%) presented with epidural abscess associated with spondylodiscitis, and 76 (74.5\%) presented with spondylodiscitis without epidural abscess (see Table 5). Patients who presented with epidural abscess $(n=26)$ were more likely to present with some degree of neurological deficits. In 1 case, the patient's impairment was classified as Grade A on the American Spinal Injury Association (ASIA) Impairment Scale (AIS), in 1 case as Grade B, in 3 cases as Grade C, and in 3 other cases as Grade D. In the 18 other cases, the AIS grade was $\mathrm{E}$ (no impairment).

\section{Other Comorbidities}

Thirty-four patents presenting with spinal infection had concomitant hepatitis C, 1 patient had HIV infection, and 2 patients had coexistent HIV and hepatitis C. Eight patients were diagnosed with endocarditis, 7 were diagnosed with with diabetes mellitus, and 12 admitted alcohol 
Spinal infections in intravenous drug users

TABLE 1: Characteristics of our study population*

\begin{tabular}{|c|c|}
\hline Characteristic & Value $(\%)$ \\
\hline no. of patients & 102 \\
\hline \multicolumn{2}{|l|}{ sex } \\
\hline male & $88(86.3)$ \\
\hline female & $14(13.7)$ \\
\hline \multicolumn{2}{|l|}{ age (yrs) } \\
\hline mean & 45.4 \\
\hline range & $22-66$ \\
\hline \multicolumn{2}{|l|}{ WBC count at presentation } \\
\hline mean $\left(\times 10^{3}\right.$ cells $\left./ \mu \mathrm{l}\right)$ & 11.1 \\
\hline range $\left(\times 10^{3}\right.$ cells $\left./ \mu \mathrm{l}\right)$ & $0.5-32$ \\
\hline no. of pts w/ abnormal WBC count & $50(49)$ \\
\hline \multicolumn{2}{|l|}{ ESR at presentation } \\
\hline mean (mm/hr) & 74 \\
\hline range (mm/hr) & $9.9-140$ \\
\hline no. of pts w/ abnormal ESR & $93(91)$ \\
\hline \multicolumn{2}{|l|}{ CRP level at presentation } \\
\hline mean $(\mathrm{mg} / \mathrm{L})$ & 67 \\
\hline range (mg/L) & $0.1-323$ \\
\hline no. of pts w/ abnormal CRP level & $73(71.6)$ \\
\hline
\end{tabular}

* Values represent numbers of patients (\%) unless otherwise indicated. $\mathrm{Pt}=$ patient.

abuse (Table 2). Nine patients admitted remote IV drug abuse (3-20 years previously) but also admitted recent use of recreational drugs via other routes and had other comorbidities, including alcohol abuse (3 patients), hepatitis C (3 patients), diabetes mellitus (1), and lymphoma (1). Of these patients one had experienced a recent fall; another was involved in a motor vehicle accident; 5 patients had undergone recent surgical procedures for dental pathology (2 patients), colon cancer (1), or splenectomy after traumatic injury (1) or ankle injections for ankle pain (1); and 2 patients had undergone remote back surgery (4 years earlier).

\section{Laboratory Data}

The average WBC count at presentation was $11.1 \times$ $10^{3}$ cells/ $\mu$ l (range $0.5-32 \times 10^{3}$ cells $/ \mu \mathrm{l}$; normal range $5.5-11 \times 10^{3}$ cells $\left./ \mu \mathrm{l}\right)$. The WBC count was in the normal range in 52 patients $(51 \%)$, while 2 patients presented with leukopenia $\left(<5.5 \times 10^{3}\right.$ cells $\left./ \mu \mathrm{l}\right)$. The mean erythrocyte sedimentation rate (ESR) and C-reactive protein (CRP) levels at presentation were $74 \mathrm{~mm} / \mathrm{hr}$ (range 9.9-140

TABLE 2: Comorbidities found in our patient cohort

\begin{tabular}{lc}
\hline Comorbidity & No. of Pts \\
\hline hepatitis C & 34 \\
EtOH abuse & 12 \\
endocarditis & 8 \\
diabetes mellitus & 7 \\
HIV & 3 \\
hepatitis B & 2
\end{tabular}

$\mathrm{mm} / \mathrm{hr}$ ) and $67 \mathrm{mg} / \mathrm{L}$ (range $0.1-323 \mathrm{mg} / \mathrm{L}$ ), respectively. Laboratory values showed that 9 patients $(8.8 \%)$ presented with normal ESR (<9.9 mm/hour) and 30 (29\%) presented with normal CRP $(<0.1 \mathrm{mg} / \mathrm{L})$ (Table 1). Due to the suboptimal compliance with follow-up visits, we were able to track the normalization of the inflammatory markers in only 44 of the 73 patients who had abnormal values at initial presentation. The average number of days from presentation with abnormal inflammatory markers to their normalization after treatment in these patients was 44.8 days (range 6-130 days).

In 40 cases $(39.2 \%)$ methicillin-sensitive Staphylococcus aureus (MSSA) grew in the culture media, while methicillin-resistant $S$. aureus (MRSA) grew in 23 (22.5\%). Only 4 patients had Pseudomonas aeruginosa isolated from the tissue of their bone biopsy. Ten patients had no growth on their cultures from either a peripheral source or bone biopsy (Table 3).

\section{Spinal Level Distribution}

The most commonly affected levels were lumbar (59 patients [57.8\%]), followed by thoracic (24 [23.5\%]) and cervical (9 [8.8\%]). The remaining patients had infections spanning either in the cervicothoracic junction or the thoracolumbar junction. A single level was involved in 71 patients $(69.6 \%)$, with the rest having involvement of multiple levels (Table 4).

The regional distribution of epidural abscesses is shown in Table 4. In 3 patients, epidural abscess expanded in multiple spine regions, involving cervicothoracic or thoracolumbar areas. The length of the epidural abscess in different patients ranged from $15 \mathrm{~mm}$ to $186 \mathrm{~mm}$, and the width within the spinal canal ranged from $4.2 \mathrm{~mm}$ to $13.8 \mathrm{~mm}$.

\section{Medical Treatment}

Eighty patients (78.4\%) underwent medical treatment with intravenous antibiotic therapy tailored to their specific organism for 6 to 8 weeks. If no organism was

TABLE 3: Organisms found in spine infections in our patient cohort*

\begin{tabular}{lc}
\hline \multicolumn{1}{c}{ Organism } & No. of Isolates \\
\hline MSSA & 40 \\
MRSA & 23 \\
streptococcal spp. (viridans, anginosus, bovis) & 6 \\
mixed culture (mainly mixed E. coli \& & 5 \\
$\quad$ Klebsiella spp.) & \\
P. aeruginosa & 4 \\
Serratia marcescens & 3 \\
Propionibacterium acnes & 3 \\
Brucella (Dx based on Brucella titers) & 2 \\
Mycobacterium (1 cholanae, 1 tuberculosis) & 2 \\
E. coli & 1 \\
Proteus mirabilis & 1 \\
no growth & 10 \\
\hline
\end{tabular}

* Dx = diagnosis. 
TABLE 4: Spinal segments involved in our study population*

\begin{tabular}{lccc}
\hline Spinal Segment & Total & Epidural Abscess Alone & $\begin{array}{c}\text { Vertebral Osteomyelitis \& } \\
\text { Epidural Abscess }\end{array}$ \\
\hline cervical & $9(8.8)$ & 1 & 0 \\
thoracic & $24(23.5)$ & 2 & 5 \\
lumbar & $59(57.8)$ & 1 & 13 \\
single level & $71(69.6)$ & 0 & 0 \\
multiple levels & $31(30.3)$ & 3 & 0 \\
\hline
\end{tabular}

* Values represent numbers of patients (\%).

identified in cultures, a 6-week course of broad-spectrum empirical antibiotic coverage was prescribed. Of the 7 patients with epidural abscess alone, only 2 (29\%) underwent medical treatment alone; the other $5(71 \%)$ underwent surgical intervention (Table 5). Of those 2 patients, 1 presented without neurological deficit (ASIA E) and the other refused surgical intervention. Twelve $(63.2 \%)$ of the 19 patients with epidural abscess associated with spondylodiscitis underwent medical treatment alone, and the rest underwent surgical intervention followed by antibiotic therapy.

\section{Surgical Treatment}

Twenty-four patients (23.5\%) required surgical intervention, 22 at presentation for acute neurological deficits, open biopsy, or spinal deformity and 2 after failure of medical therapy (Fig. 1). Eight patients (36.3\%) underwent laminectomy alone for epidural abscess, 6 underwent open biopsy, 7 underwent anterior vertebrectomy with either anterior instrumentation alone or combined anterior and posterior instrumentation, and 3 underwent laminectomy and posterior instrumentation. Five of the 7 patients who presented with epidural abscess alone underwent laminectomy or laminotomy because of presentation with acute neurological deficit.

\section{Follow-Up and Outcome}

The average duration of follow-up for the patients who underwent intervention was 29.7 weeks (range 6 weeks-3 years). Follow-up evaluation at 6 weeks after initiation of treatment was performed in 83 cases $(81 \%)$. The 83 patients in these cases all remained in the hospital for the entire duration of the IV antibiotic treatment. At 3 months, 79 patients $(78 \%)$ presented at the follow-up visits; at 12 months, 72 patients (70.5\%); and only 66 (64.7\%) presented for follow-up visits at their primary care physician's office after the 1-year mark (up to 3 years) (Table
6). The infectious diseases consultant evaluated all patients, and their course was followed with clinical assessment and serial laboratory tests (ESR and/or CRP level) if they presented for follow-up and had shown elevated inflammatory markers at initial presentation. Due to the accepted concept that follow-up imaging is not warranted in all patients because improvement of the MRI appearance lags behind improvement in clinical presentation, 3,9 we reserved imaging studies for patients who did not demonstrate clinical improvement.

Two patients who had previously undergone surgical intervention presented with osteomyelitis at the adjacent levels and required reoperation with extension of the arthrodesis and posterior instrumentation. Both of these patients had continued their IVDA habits. Three patients had superficial wound infections; 2 were treated with wound care and a new course of IV antibiotic therapy, and 1 underwent surgical debridement. A significant number of patients in our cohort had multiple "no-show" appointments. In the nonsurgically managed group the average duration of follow-up was 6 months. None of the patients had worsening of their neurological condition during the follow-up period. Two patients who required surgical intervention due to failure of medical therapy presented with increased back pain (Fig. 1). Neither of these patients had epidural abscess at first presentation.

\section{Discussion}

Although recreational IVDA is thought to be one of the major factors in the recent increase in the incidence of spinal infection, very few reports have described exclusively this in the literature. Sapico and Montgomerie ${ }^{18}$ reported 3 cases of spine infection in IVDA patients and identified 64 other cases reported in the literature. They found that the majority of patients had lumbar spine involvement $(53.5 \%)$ and with respect to causative agents

TABLE 5: Three pathological entities and the stratification of patients based on medical and surgical management*

\begin{tabular}{lccc}
\hline \multicolumn{1}{c}{ Pathological Entity } & Total & Treated Medically & Treated Surgically \\
\hline vertebral osteomyelitis \& epidural abscess & $19(18.6)$ & 12 & 7 \\
epidural abscess alone & $7(6.9)$ & 2 & 5 \\
vertebral osteomyelitis alone & $76(74.5)$ & 64 & 12 \\
total & 102 & 78 & 24 \\
\hline
\end{tabular}

* Values represent numbers of patients (\%). 

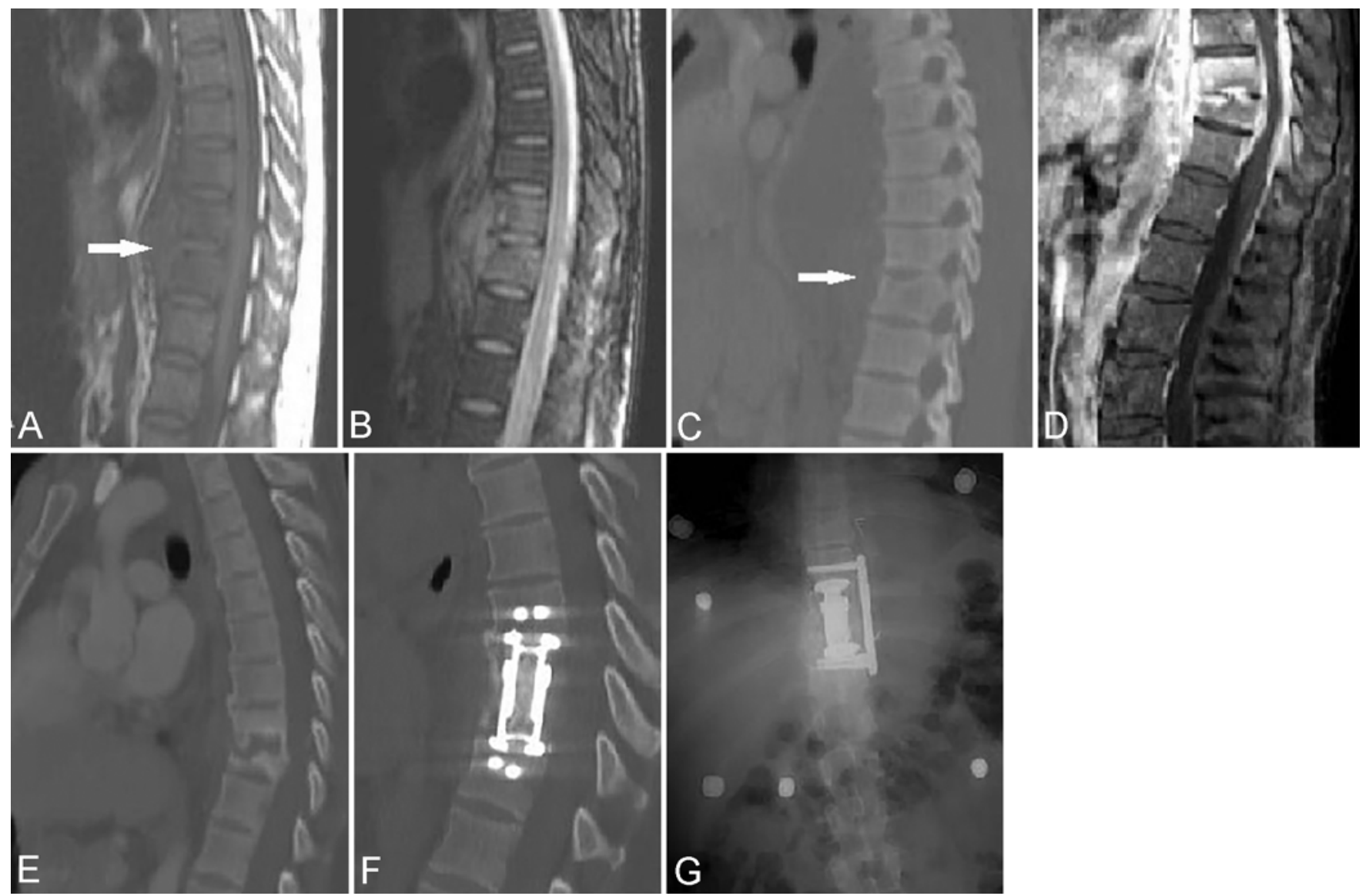

Fig. 1. Example of a 46-year-old man with a history of heroin abuse and a 1-month history of thoracic back pain in whom medical therapy failed. No neurological deficits were noticed on examination. MRI and CT at presentation showed spondylodiscitis $(\mathrm{A}-\mathrm{C})$, and needle biopsy cultures grew MRSA. The WBC count was $15.9 \times 10^{3} \mathrm{cells} / \mathrm{\mu l}$, the ESR was $40 \mathrm{~mm} / \mathrm{hr}$, and the CRP level was $314 \mathrm{mg} / \mathrm{L}$. The patient was discharged on 6 weeks of IV vancomycin treatment and with normalizing laboratory values. He returned 5 weeks later with complaints of increased back pain and tingling in the lower extremities. Imaging (D and E) demonstrated progressive kyphosis. He underwent anterior vertebrectomy, cage placement, and anterior instrumented arthrodesis ( $F$ and $\mathbf{G})$, and an additional 6 weeks of intravenous antibiotic therapy. At his last follow-up visit, 3 months postoperatively, he was doing well.

reported that the $65.5 \%$ of all isolates were Pseudomonas (labeled "Pseudomonas species" or P. aeruginosa). Chuo et al. ${ }^{5}$ reported 21 cases of spine infection in IV drug abusers, with 19 suffering from pyogenic spine infection. Wang and colleagues have reported the only prospective study performed to date where diagnosis, management, and outcome of 51 patients with spinal infection and IVDA was compared with a cohort of patients with spinal infection without recreational IV drug use. ${ }^{19}$

We report here on a cohort of 102 consecutive recreational patients with a history of IVDA who were treated for spinal infection at our hospital. To our knowledge,

TABLE 6: Duration of clinical follow-up

\begin{tabular}{cc}
\hline Duration of Follow-Up & No. of Pts (\%) \\
\hline 6 wks & $83(83)$ \\
3 mos & $79(78)$ \\
12 mos & $72(71)$ \\
$>1 \mathrm{yr}$ & $66(65)$ \\
\hline
\end{tabular}

ours is the largest series of patients with spine infection and IVDA as the major risk factor. The majority of our patients were male, aged 41-60 years, and had predominantly lumbar localization $(57.8 \%)$, similar to the findings of Sapico and Montgomerie ${ }^{18}$ and Chuo et al. ${ }^{5}$ Our data, however, differ from the finding of Wang et al., who reported predominantly cervical spine involvement. ${ }^{19}$ The difference for these findings could be explained by the location of injection in the cases reported by Wang and colleagues, whose patients used jugular veins more frequently.

Illicit drug abuse is a worldwide health problem. Approximately 5\% of the global population (200 million people) use illicit drugs. ${ }^{6}$ It has been reported that there are an estimated 13 million injection-drug users worldwide, $78 \%$ of whom live in developing countries. ${ }^{6}$ Unfortunately infections, such as HIV, viral hepatitis, and various bacterial infections, are among the most serious complications of IV drug use. According to a review by Gordon and Lowy, ${ }^{6}$ most bacterial infections in this population are caused by the individual's own commensal flora, with S. aureus and Streptococcus species being 
the most common pathogens isolated. Infection by organisms such as $P$. aeruginosa indicates that a specific drug or drug-use behavior is involved. ${ }^{6}$ In agreement with the findings of Chuo et al. ${ }^{5}$ and Wang et al., ${ }^{19}$ we found that in the majority of our patients, the causative organisms was S. aureus-MSSA in $39.2 \%$ and MRSA in $22.5 \%$ of the patients (Table 2). Pseudomonas aeruginosa was found only in 4 patients $(3.9 \%)$ in our series, a finding that contrasts with that of Sapico and Montgomerie who determined that Pseudomonas species were isolated in $65.5 \%$ of the 64 cases they identified in their literature review. ${ }^{18}$ Musculoskeletal infections generally result from hematogenous seeding or local extension of a skin or softtissue infection in drug users. These infections may be indolent, with the only symptom being pain without fever. ${ }^{6}$ All of our 102 patients had severe back and/or neck pain at presentation, suggesting that a history of recent or remote recreational IVDA associated with back pain should prompt further diagnostic workup, preferably MRI with and without contrast, to exclude spinal infection even in the absence of fever and abnormal WBC count.

In the IVDA population, in contrast to the general population, diabetes mellitus does not appear to be a factor that predisposes patients to primary pyogenic spinal infection. Only 7 patients $(6.9 \%)$ in our population had diabetes mellitus. Hepatitis $\mathrm{C}$ positivity was found in 34 patients (33\%), hepatitis B in 2, and HIV in 3 patients. We have to consider that the nonsterile practice of use of needles for drug injection and needle interchange with other individuals predisposes these patients to multiple infectious agents. Endocarditis was found in only 8 patients, suggesting that hematogenous seeding of bacteria in the spine is not related to the presence of endocarditis and septic emboli.

The diagnosis of spinal infection in patients with neck or back pain and a history of IVDA requires a high index of suspicion. The experience from our institution shows that routine laboratory tests are poor markers for this diagnosis. The WBC counts were elevated on admission in only $49 \%$ of our patients; ESR and CRP levels (inflammatory markers) were increased in $91 \%$ and $71 \%$ of the patients, respectively. These laboratory values-if abnormal-were sensitive indicators of the disease progression or regression during treatment (data not shown), although they are not specific for initial diagnosis of spine infection in IVDA patients.

Figure 2 presents the management flowchart developed and applied in our institution. When a patient with back pain and a history of IVDA presents to our hospital, an MRI study with and without contrast is requested, and if spinal infection is suspected from the MRI findings, infection disease specialists and spine surgeons are consulted. At the same time laboratory evaluation of WBC count, ESR, and CRP level is performed in conjunction with blood and urine cultures. The patient is furthermore evaluated for the presence of any other site of infection. Two patients in our cohort were found to have other joint infections, one in a hip and the other in a knee joint.

All our patients undergo a full evaluation by infection disease specialists to find the source and/or other sites of infection. All patients are initially treated with antibiotic therapy based on culture results for a duration of 6 to 8 weeks. In cases in which surgical intervention is not indicated, image-guided biopsy of the suspicious spine lesion is performed. Surgical intervention is considered only in the event of medical therapy failure revealed by progression of the spine deformity, new neurological deficit or progression of old neurological deficits, persistent or worsening pain, and persistent elevation of CRP and ESR values, in combination with worsening of findings on MRI, and when image-guided needle biopsy is negative.

The main pillar for treatment of spine infection even in the IVDA population remains antibiotic therapy, as in the non-IVDA population. Only 24 of our patients underwent a surgical procedure. The other 78 patients $(76 \%)$ in our series were treated exclusively with IV antibiotic therapy, and over a mean follow-up period of 6 months they showed improvement of back and/or neck pain and neurological status, confirming that surgical intervention is needed only in selected cases. This approach is in line with what others have suggested in the literature.,17 $\mathrm{Pa}$ tients with acute neurological deficits secondary to the lesion found in the MRI and those with newly diagnosed spine deformity caused by spondylodiscitis are considered for surgical intervention. Resai and colleagues ${ }^{17}$ have suggested that patients with kyphotic deformity of more than $11^{\circ}$ in the cervical spine or $20^{\circ}$ in the thoracolumbar spine should be considered for surgical intervention and deformity correction. They caution, however, that these suggestions are extrapolated from guidelines for instability in spine trauma because such studies for kyphosis in spine infection do not exist. ${ }^{17}$

Regarding the presence of epidural abscess, 5 of the 7 patients who underwent surgical decompression presented with acute neurological deficits. In our cohort, 26 patients presented with epidural abscess alone (7 patients) or in association with spondylodiscitis (19 patients). Only 12 patients (46\%) from this group underwent surgical intervention followed by antibiotic therapy, 8 due to acute neurological deficit (AIS Grade A, B, C, or D at presentation) and 4 others due to spondylodiscitis induced spinal deformity (AIS Grade E at presentation). The other 14 patients with epidural abscess and/or spondylodiscitis without acute neurological deficit or spinal deformity were treated with antibiotic therapy alone. Our data suggest that antibiotic therapy alone should be considered as the first line of treatment in patients with epidural abscess without acute neurological deficit and/or severe spinal deformity and that in those patients, epidural abscess should not be considered a neurosurgical emergency.

Although our patients' compliance was not optimal, we believe that $71 \%$ follow-up compliance at 1 year is an acceptable value for this patient population. Failure of antibiotic therapy was seen in only 2 patients $(1.9 \%)$ treated with antibiotics alone and 2 other patients treated with surgical intervention followed by antimicrobial therapy. None of the patients who had epidural abscess and were treated with medical therapy alone developed any new neurological deficit or spinal deformity while under treatment. Nevertheless, we recommend caution in interpreting these data due to the suboptimal long-term follow-up rate. 


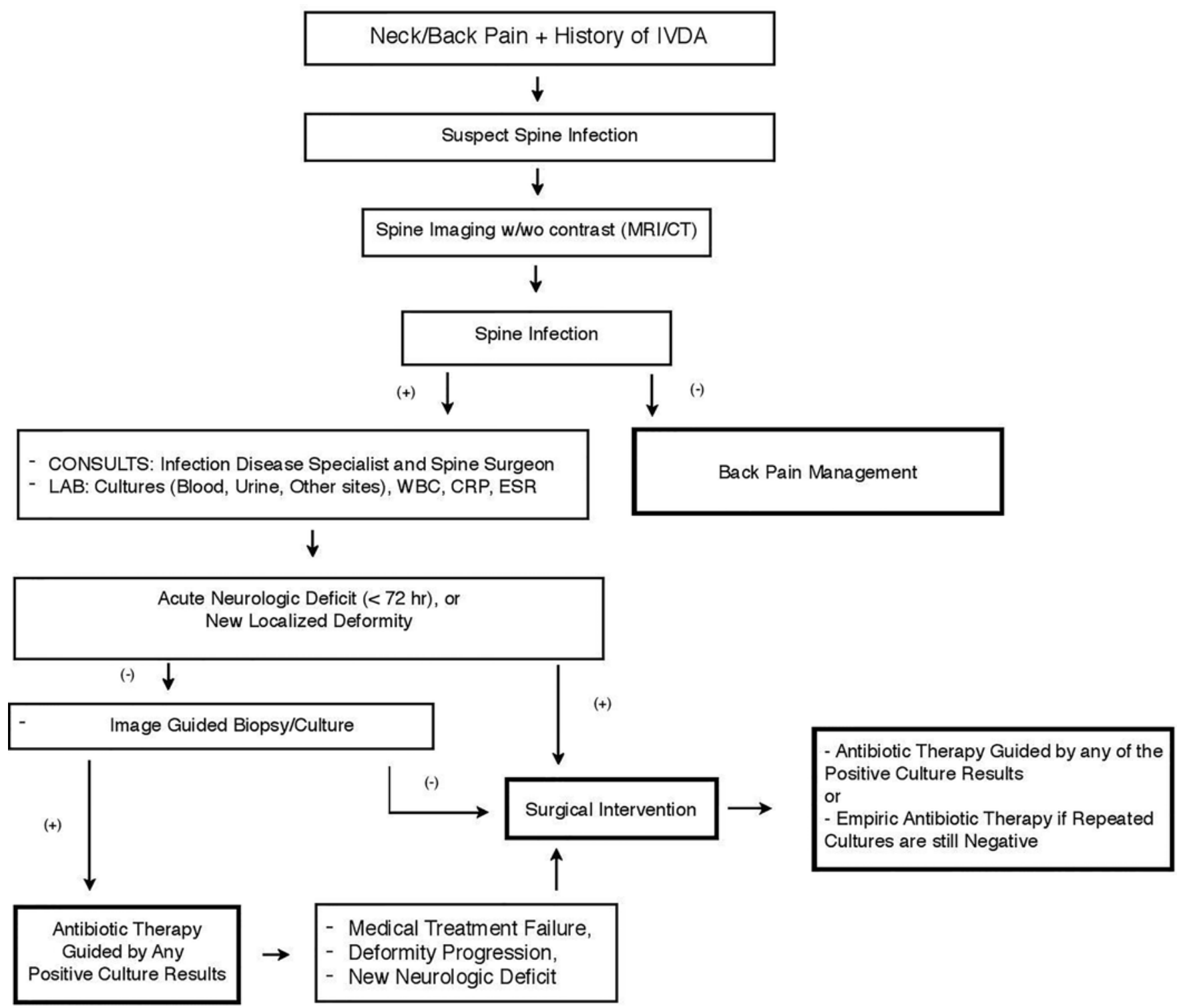

FIG. 2. Algorithm for diagnosis and management of spine infection of patients with IVDA in our institution.

In our series of 102 patients, the patients who underwent surgical intervention did so mainly for progressive kyphosis while on antibiotic therapy, acute neurological deficits secondary to epidural abscess, or newly diagnosed severe infection-induced kyphotic deformity at presentation.

\section{Conclusions}

The treatment of spinal infection in the IVDA population is difficult and further complicated by the fact that many of these patients will continue to use recreational drugs even after diagnosis has been made and the treatment has been started. Furthermore, this patient population has shown to be noncompliant with treatment protocols and follow-up. Their discharge from the hospital is complicated by the concern for their safety as the IV access used for long-term antibiotic therapy could be abused for drug self-injection. We conclude that any patient with a history of IVDA presenting with back pain should undergo imaging (preferably MRI with and without contrast) to evaluate for a possible spine infection even when the results of laboratory tests for inflammatory parameters are within the normal range. The majority of these patients can be effectively treated with antibiotics alone, with surgical intervention being necessary only in a minority of cases.

\section{Disclosure}

The authors report no conflict of interest concerning the materials or methods used in this study or the findings specified in this paper.

Author contributions to the study and manuscript preparation include the following. Conception and design: Ziu, Bartanusz. Acquisition of data: Ziu, Dengler, Cordell. Analysis and interpretation of data: Ziu, Dengler, Bartanusz. Drafting the article: Ziu, Dengler. Critically revising the article: Ziu, Dengler, Bartanusz. 
Reviewed submitted version of manuscript: all authors. Approved the final version of the manuscript on behalf of all authors: Ziu. Statistical analysis: Ziu, Dengler, Bartanusz. Administrative/technical/ material support: Ziu. Study supervision: Ziu, Bartanusz.

\section{References}

1. Beronius M, Bergman B, Andersson R: Vertebral osteomyelitis in Göteborg, Sweden: a retrospective study of patients during 1990-95. Scand J Infect Dis 33:527-532, 2001

2. Butler JS, Shelly MJ, Timlin M, Powderly WG, O'Byrne JM: Nontuberculous pyogenic spinal infection in adults: a 12-year experience from a tertiary referral center. Spine (Phila Pa 1976) 31:2695-2700, 2006

3. Carragee EJ: The clinical use of magnetic resonance imaging in pyogenic vertebral osteomyelitis. Spine (Phila Pa 1976) 22: 780-785, 1997

4. Chelsom J, Solberg CO: Vertebral osteomyelitis at a Norwegian university hospital 1987-97: clinical features, laboratory findings and outcome. Scand J Infect Dis 30:147-151, 1998

5. Chuo CY, Fu YC, Lu YM, Chen JC, Shen WJ, Yang CH, et al: Spinal infection in intravenous drug abusers. J Spinal Disord Tech 20:324-328, 2007

6. Gordon RJ, Lowy FD: Bacterial infections in drug users. $\mathbf{N}$ Engl J Med 353:1945-1954, 2005

7. Hsieh PC, Wienecke RJ, O'Shaughnessy BA, Koski TR, Ondra SL: Surgical strategies for vertebral osteomyelitis and epidural abscess. Neurosurg Focus 17(6):E4, 2004

8. Jevtic V: Vertebral infection. Eur Radiol 14 Suppl 3:E43E52, 2004

9. Kowalski TJ, Berbari EF, Huddleston PM, Steckelberg JM, Osmon DR: Do follow-up imaging examinations provide useful prognostic information in patients with spine infection? Clin Infect Dis 43:172-179, 2006

10. Krogsgaard MR, Wagn P, Bengtsson J: Epidemiology of acute vertebral osteomyelitis in Denmark: 137 cases in Denmark 1978-1982, compared to cases reported to the National Patient Register 1991-1993. Acta Orthop Scand 69:513-517, 1998

11. Mann S, Schütze M, Sola S, Piek J: Nonspecific pyogenic spondylodiscitis: clinical manifestations, surgical treatment, and outcome in 24 patients. Neurosurg Focus 17(6):E3, 2004

12. Nolla JM, Ariza J, Gómez-Vaquero C, Fiter J, Bermejo J, Valverde J, et al: Spontaneous pyogenic vertebral osteomyelitis in nondrug users. Semin Arthritis Rheum 31:271-278, 2002

13. O'Daly BJ, Morris SF, O'Rourke SK: Long-term functional outcome in pyogenic spinal infection. Spine (Phila Pa 1976) 33:E246-E253, 2008

14. Osenbach RK, Hitchon PW, Menezes AH: Diagnosis and management of pyogenic vertebral osteomyelitis in adults. Surg Neurol 33:266-275, 1990

15. Patzakis MJ, Rao S, Wilkins J, Moore TM, Harvey PJ: Analysis of 61 cases of vertebral osteomyelitis. Clin Orthop Relat Res 1991:178-183, 1991

16. Quiñones-Hinojosa A, Jun P, Jacobs R, Rosenberg WS, Weinstein PR: General principles in the medical and surgical management of spinal infections: a multidisciplinary approach. Neurosurg Focus 17(6):E1, 2004

17. Rezai AR, Woo HH, Errico TJ, Cooper PR: Contemporary management of spinal osteomyelitis. Neurosurgery 44:10181026, 1999

18. Sapico FL, Montgomerie JZ: Vertebral osteomyelitis in intravenous drug abusers: report of three cases and review of the literature. Rev Infect Dis 2:196-206, 1980

19. Wang Z, Lenehan B, Itshayek E, Boyd M, Dvorak M, Fisher C, et al: Primary pyogenic infection of the spine in intravenous drug users: a prospective observational study. Spine (Phila Pa 1976) 37:685-692, 2012

Manuscript submitted April 16, 2014.

Accepted June 11, 2014.

Current affiliation for Dr. Cordell: Department of Orthopedics at University of Texas Health Science Center in San Antonio, Texas.

Please include this information when citing this paper: DOI: 10.3171/2014.6.FOCUS14148.

Address correspondence to: Mateo Ziu, M.D., Seton Brain and Spine Institute, 1400 N. I-35, Ste. 300, Austin, TX 78701. email: mziu@seton.org. 